\title{
Generation and Trapping of Gravity Waves from Convection with Comparison to Parameterization
}

\author{
M. JoAn Alexander \\ Colorado Research Associates Division, NorthWest Research Associates, Boulder, Colorado \\ JADWigA H. (BERES) RichteR \\ National Center for Atmospheric Research, Boulder, Colorado \\ BRUCE R. SUTHERLAND \\ University of Alberta, Edmonton, Alberta, Canada
}

(Manuscript received 3 June 2005, in final form 21 March 2006)

\begin{abstract}
Some parameterizations of gravity wave mean flow forcing in global circulation models (GCMs) add realism by describing wave generation by tropospheric convection. Because the convection in GCMs is itself a parameterized process, these convectively generated wave parameterizations necessarily use many simplifying assumptions. In this work, the authors use a realistic simulation of wave generation by convection described in previous work, which was validated by observations from the Darwin Area Wave Experiment (DAWEX), to test these assumptions and to suggest some possible improvements to the parameterizations. In particular, the authors find that wave trapping in the troposphere significantly modifies the spectrum of vertically propagating waves entering the stratosphere above convective wave sources, and offer a linear method for computing wave transmission and reflection effects on the spectrum suitable for inclusion in the parameterizations. The wave fluxes originate from both a time-varying heating mechanism and an obstacle effect mechanism acting in the simulation. Methods for including both mechanisms in the parameterizations are described. Waves emanating from the obstacle effect remain very sensitive to the depth of penetration of latent heating cells into an overlying shear zone, which will continue to make it difficult to accurately parameterize in a GCM where the convective cells are not resolved.
\end{abstract}

\section{Introduction}

Convection is known to generate atmospheric waves of all scales from planetary-scale (Matsuno 1966; Holton 1972; Lindzen 1974; Salby and Garcia 1987; Bergman and Salby 1994; Wheeler and Kiladis 1999) to small-scale, high-frequency gravity waves (Fovell et al. 1992; Dewan et al. 1998; Piani et al. 2000). The gravity waves, in general, are too small to be resolved in global models: yet collectively these waves are known to provide significant forcing of tropical global-scale circulations. In the lower stratosphere, they help to drive the quasi-biennial oscillation in zonal winds (Dunkerton

Corresponding author address: Dr. M. J. Alexander, Colorado Research Associates Division, NorthWest Research Associates, 3380 Mitchell Lane, Boulder, CO 80301.

E-mail: alexand@cora.nwra.com
1997; Giorgetta et al. 2002) and, at higher altitudes, they contribute to the stratospheric and mesospheric semiannual oscillations. The effects of the gravity waves must currently be parameterized in global models, and recent attempts to parameterize convectively generated gravity waves have shown some interesting results (Chun et al. 2004; Beres et al. 2005). However, these parameterizations are poorly constrained by observations at present. The parameterizations are by necessity simple and are based on the linear wave response to localized and/or time-varying latent heating (Chun and Baik 1998, 2002; Beres et al. 2004). Although nonlinear effects are known to be important (Song et al. 2003), one must hope that the parameterizations can be modified or corrected somehow to approximately account for these effects in order to continue to make progress in the global modeling arena. This is one of the goals of the present work. 
As part of the Darwin Area Wave Experiment (DAWEX), we previously conducted simulations of wave generation by convection using precipitation radar reflectivity to define the wave forcing in a dry version of a nonlinear cloud-resolving model (Alexander et al. 2004). We further compared the waves in this model to locally observed wave features in radiosondes (Tsuda et al. 2004) and to real-time observations of wave structures in boundary layer radar observations (Vincent et al. 2004) taken during the campaign. The results of the comparison validated the technique for describing waves generated by convection. Previous modeling studies of wave generation by convection have used cloud-resolving models with microphysical parameterizations (Fovell et al. 1992; Alexander and Holton 1997; Piani et al. 2000; Lane and Reeder 2001). Although these models have been useful tools for understanding wave generation by convection, the convection in these models is only qualitatively similar to observations. The rain cells do not occur at the correct place or at the correct time. They can only be compared to observations in some qualitative or statistical manner, but one would never expect a boundary layer radar time series of wave motions to resemble the time series at the same location in the model, as was shown in the Vincent et al. (2004) study. In comparison, our model results showed some very realistic features in both the characteristics of the waves observed by the radar and the timing of their occurrence (Vincent et al. 2004).

In the present paper, we use the Alexander et al. (2004) simulation in a comparison to a parameterization of wave generation that describes the spectrum of vertically propagating waves emanating from convection (Beres 2004). This model/parameterization set is particularly suitable because both depend on the horizontal and vertical scales and the timing and duration of the latent heating in convective clouds. The model resolves the structure of the latent heating and waves in a $400 \mathrm{~km} \times 400 \mathrm{~km}$ time-dependent domain in a case that has been well validated by observations. Parameterizations, in contrast, must assume single simple properties of the heat sources within the global model grid box, an area similar to our full model domain. We can also use the model to examine the effects of background wind shear on the wave spectrum while maintaining constant latent heating. The Beres (2004) parameterization, in contrast, must assume a constant wind at all altitudes where the latent heating is nonzero, so we examine the effects of this assumption on the parameterized wave spectrum.

The primary effect of the wind will be shown to be the interaction of the upper-tropospheric wind at the tops of the latent heating cells. The mechanism has been previously described as the "obstacle effect" (Clark et al. 1986; Pfister et al. 1993; Beres et al. 2002). It was the only process included in the Chun and Baik parameterization (Chun and Baik 1998, 2002). The Beres (2004) parameterization, conversely, focused on the high phase speed wave response and neglected the obstacle effect because the vertically propagating wave fluxes were extremely sensitive to the wind shear profile and the exact height to which the latent heating cell penetrates (Beres et al. 2002, 2004; Chun and Baik 2002). Global models can only provide heating depths and shear in a grid-box-average sense, whereas the wave generation will be sensitive to the depth of localized small-scale heating cells and the shear they encounter aloft. The flux for a single average cell will often not predict well the average of an ensemble of cells. Gravity wave parameterizations in global models describe the heating with a single set of parameters for each grid box, and the resulting momentum fluxes from the obstacle effect would be highly uncertain. In contrast, in our nonlinear convectively generated wave simulation, the individual heating cells are resolved and the average obstacle effect can be exactly quantified within our model simulations. We can then suggest a method to parameterize the obstacle effect, at least for this one DAWEX case study that is fairly well validated. Additional studies will be needed to generalize these results before the parameterizations can describe the wave generation from various types of convection in the Tropics with any accuracy.

We also examine the effects of wave trapping on the vertically propagating wave spectrum in both the model and parameterization. Although the importance of trapped waves generated by convection to storm structure (Yang and Houze 1995) and storm initiation (Mapes 1993; Shige 2001) has been described in the literature, the effects of wave trapping on the vertically propagating wave spectrum have so far been neglected in convectively generated wave parameterizations. In this work we describe an approximate (linear) description of wave trapping effects that explains most of the features of trapped waves observed in our nonlinear simulation of gravity waves generated by latent heating in convection. This linear wave trapping model is suitable for inclusion in gravity wave parameterizations. We compute the trapping effects on the vertically propagating wave spectrum from the Beres (2004) parameterization and show that inclusion of these effects brings the parameterized spectrum much closer to the one calculated in our nonlinear simulation.

We further examine how nonlinearities affect the vertically propagating wave spectrum in the model since the parameterization is by necessity based on 
simple linear theory. The results show a systematic skewing of the spectrum as nonlinearities grow, such that the lower phase speed waves are proportionately overestimated in the more linear models, whereas the high phase speed waves are relatively unaffected. These results are qualitatively similar to Song et al. (2003), which compared a purely linear simulation to a fully nonlinear simulation. Our additional calculations suggest some possible simple ways to correct for these nonlinearity effects in the parameterization.

\section{Mesoscale model simulations}

In the present paper, we show results from a simulation described in detail in Alexander et al. (2004) plus two additional simulations that differ only in the background wind profile. These additional simulations will be used to clarify the role of the background wind in wave generation and wave propagation. Our mesoscale model and method of forcing with observations from precipitation radar are described in detail in the previous work (Alexander et al. 2004). Here we summarize only a few essential details. The model is that of Durran and Klemp (1983) with more recent modifications as described in Piani et al. (2000) and Beres et al. (2002). In the present application, we turn off all moist processes in the model and, instead, provide wave forcing with observed spatial and temporal variations in convective latent heating derived from precipitation radar (Keenan et al. 1998; May et al. 2002). The radar observations provide three-dimensional volumetric retrievals of reflectivity with $2-\mathrm{km}$ horizontal resolution, 1-km vertical resolution, and 10-min time resolution. The method of conversion between the radar reflectivity and the latent heating input to the model appears in Alexander et al. (2004). As in this previous work, we simplify the vertical profile of the deep convective heating in the conversion to a half-sine shape.

For the simulation, a horizontally uniform background atmosphere was assumed with vertical variations derived from the 5-day mean of 3-hourly DAWEX radiosonde profiles for the November campaign period. The time averaging removes largeamplitude wave oscillations that appear in the upper troposphere in individual soundings. The heating was input in a circular area $256 \mathrm{~km}$ in diameter centered on the radar site, and this was centered within the $400-\mathrm{km}-$ squared mesoscale model domain. The top and the four sides of the domain have wave permeable boundary conditions (Durran et al. 1993). The model was run at the same 2-km horizontal resolution as the radar data but at higher $0.25-\mathrm{km}$ vertical resolution to resolve the important wave responses. The radar reflectivity is re- trieved up to $19-\mathrm{km}$ altitude, and the model top is 25 $\mathrm{km}$. The buoyancy frequency $N$ profile is shown in Alexander et al. (2004) and has a tropopause at $17 \mathrm{~km}$ with constant $N=0.027 \mathrm{~s}^{-1}$ in the stratosphere and variable $N$ in the troposphere (average $N \sim 0.011 \mathrm{~s}^{-1}$ between 0 and $12 \mathrm{~km}$, a layer of low stability $N \sim 0.007$ $\mathrm{s}^{-1}$ at $12-15 \mathrm{~km}$, and then a rapid increase to stratospheric values above $15 \mathrm{~km}$ ). Because of uncertainties in the humidity profiles in the DAWEX soundings at upper levels, we use the dry static stability profile rather than the moist static stability (Durran and Klemp 1982). Using the existing data, we estimate that moisture effects might lower the stability in the upper troposphere by $\sim 10 \%-20 \%$.

\section{Spectral analysis of stratospheric waves}

The top panels in Fig. 1 show a spectrum of stratospheric wave momentum flux from the first $4 \mathrm{~h}$ of the simulation, along with vertical profiles of zonal and meridional wind for this case, which are identical to Alexander et al. (2004). Phase speed $c$ is defined here and throughout the paper as the ground-based horizontal phase speed. The contours in Fig. 1 are logarithmic to show the range of amplitudes and phase speeds in the model. The spectrum is computed from a threedimensional Fourier transform in the horizontal plane $(x, y)$ and time $t$ with $\Delta t=3 \mathrm{~min}$. The spectrum is then rebinned into horizontal phase speed $c$ and propagation direction $\phi$ and integrated over horizontal wavenumber. The results are computed at each altitude $z$ in the model stratosphere and averaged over $z=21-24 \mathrm{~km}$. [The units are of momentum flux per unit phase speed $\Delta c=2 \mathrm{~m} \mathrm{~s}^{-1}$ for later comparison to the Beres (2004) parameterization. Values of the area-averaged momentum flux will be described in the discussion section.] The most prominent result for this previously reported case is the strong response for waves propagating in the northeast (NE) direction with slow phase speeds, $\sim 5-$ $10 \mathrm{~m} \mathrm{~s}^{-1}$. This spectrum also shows the effect of wind filtering via the arc of low wave fluxes centered on the wind direction. (The stratospheric wind speed as a function of direction is also overplotted as a dashed line.)

The middle panels show the same result for a simulation with only the tropospheric portion of the background modified to approximately zero winds below 10 $\mathrm{km}$, as shown center right. The spectrum for this second case is nearly identical to the first, indicating that the winds in the troposphere below $10 \mathrm{~km}$ have virtually no effect on the stratospheric wave spectrum. The bottom panels in Fig. 1 show a third simulation, this time with constant near-zero winds at all altitudes. The wave spectrum in this case is dramatically altered. The spectrum is now nearly isotropic. The peak in fluxes at low 

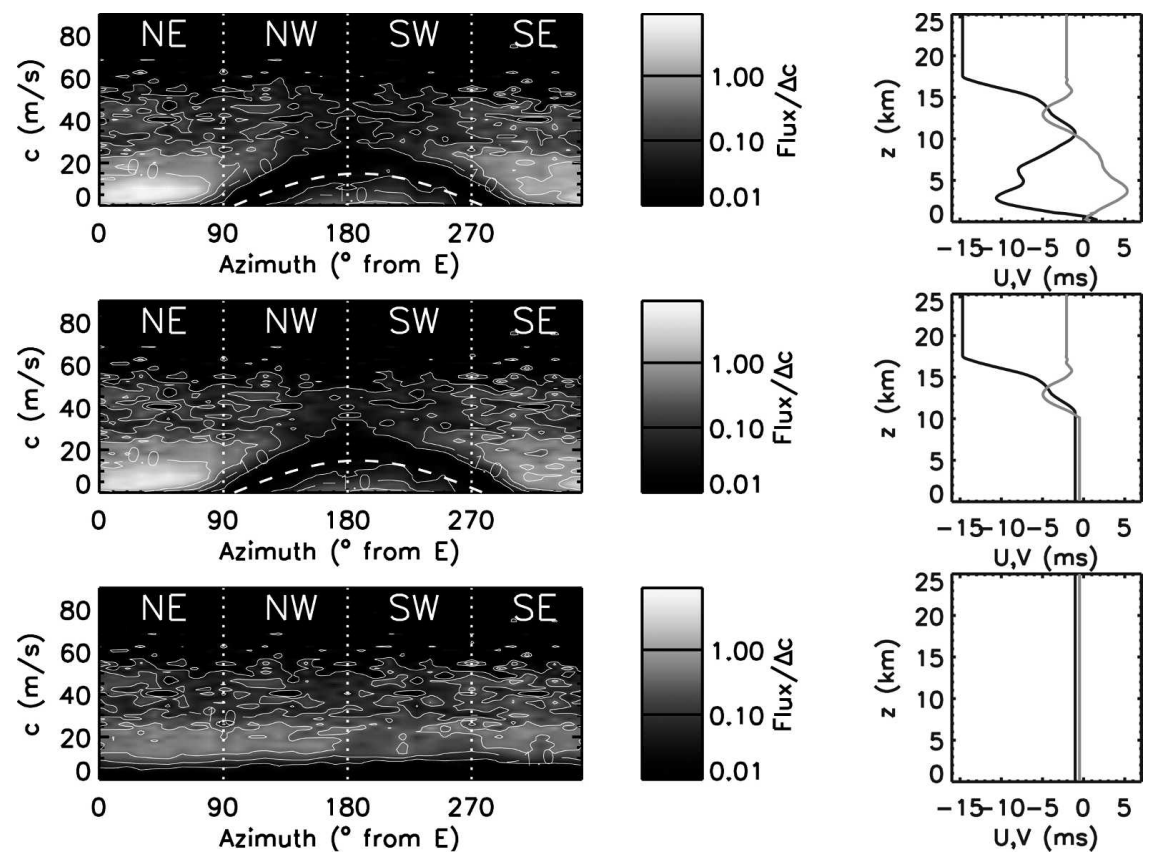

FIG. 1. Wave momentum flux vs propagation direction and ground-based phase speed for (left) the three simulations described in the text and (right) their associated wind profiles with zonal wind $U$ in black and meridional wind $V$ in gray. The dashed white line in the top two panels shows the vector stratospheric wind that serves to filter an arc-shaped region of phase speeds. Flux units are given per unit phase speed, $10^{-6} \mathrm{~Pa}\left(\mathrm{~m} \mathrm{~s}^{-1}\right)^{-1}\left(\right.$ or $\left.10^{-6} \mathrm{~kg} \mathrm{~m}^{-2} \mathrm{~s}^{-1}\right)$.

NE phase speeds has disappeared, and the arc of wind filtering is also gone.

These results clearly indicate that the wind shear between 10 and $17 \mathrm{~km}$ is of primary importance to the shape of the resulting wave spectrum and that the horizontal winds below that level have minimal effect. The results also indicate that the prominent peak in momentum flux at low phase speeds in the NE direction is generated by the upper-tropospheric winds interacting with the latent heating sources. This is wave generation by the so-called obstacle effect. If we can quantify the effects of wind filtering on the spectrum, we can then quantify the wave generation via the obstacle effect by examining the differences between the simulations in the middle and bottom panels in Fig. 1. In the next section, we quantify the filtering and reflection effects of the background winds on the vertically propagating wave spectrum.

\section{Wave trapping and wind filtering effects on the vertically propagating wave spectrum}

Examination of the model vertical winds in the troposphere reveals trapped waves with a southeasterly preference in their propagation direction (Fig. 2). Figure 2 is taken from the full wind simulation (top panel in Fig. 1), but the trapped waves are very similar in the simulation with only upper-tropospheric wind shear (middle panel in Fig. 1). Trapped waves generated by convection have been implicated in remote initiation of subsequent convection in model studies (Mapes 1993; Shige 2001). In the two-dimensional tropical squall line model study of Shige (2001), a preference for westward trapped wave propagation was found. The upper-level shear in their model was westward, similar to our case study. The authors suggested that the westward preference was caused by wave trapping due to an overreflection mechanism (Lindzen and Tung 1976) that can occur as waves approach critical levels (where the mean wind speed in the direction of propagation approaches c). In our study, we find a preference for trapping of waves propagating in the opposite direction, toward the east. The trapping in our case is best described by internal reflection that instead occurs for waves with high $c$ propagating upstream from the background wind (Lighthill 1978; Marks and Eckermann 1995).

Trapped waves carry no net momentum flux vertically, so the cospectra of horizontal and vertical wind perturbations $\left(\overline{u^{\prime} w^{\prime}}, \overline{v^{\prime} w^{\prime}}\right)$ associated with these waves in the model are nearly zero. To examine the properties of the trapped waves, we look at the power spectrum of vertical velocity in the troposphere. To see the spec- 

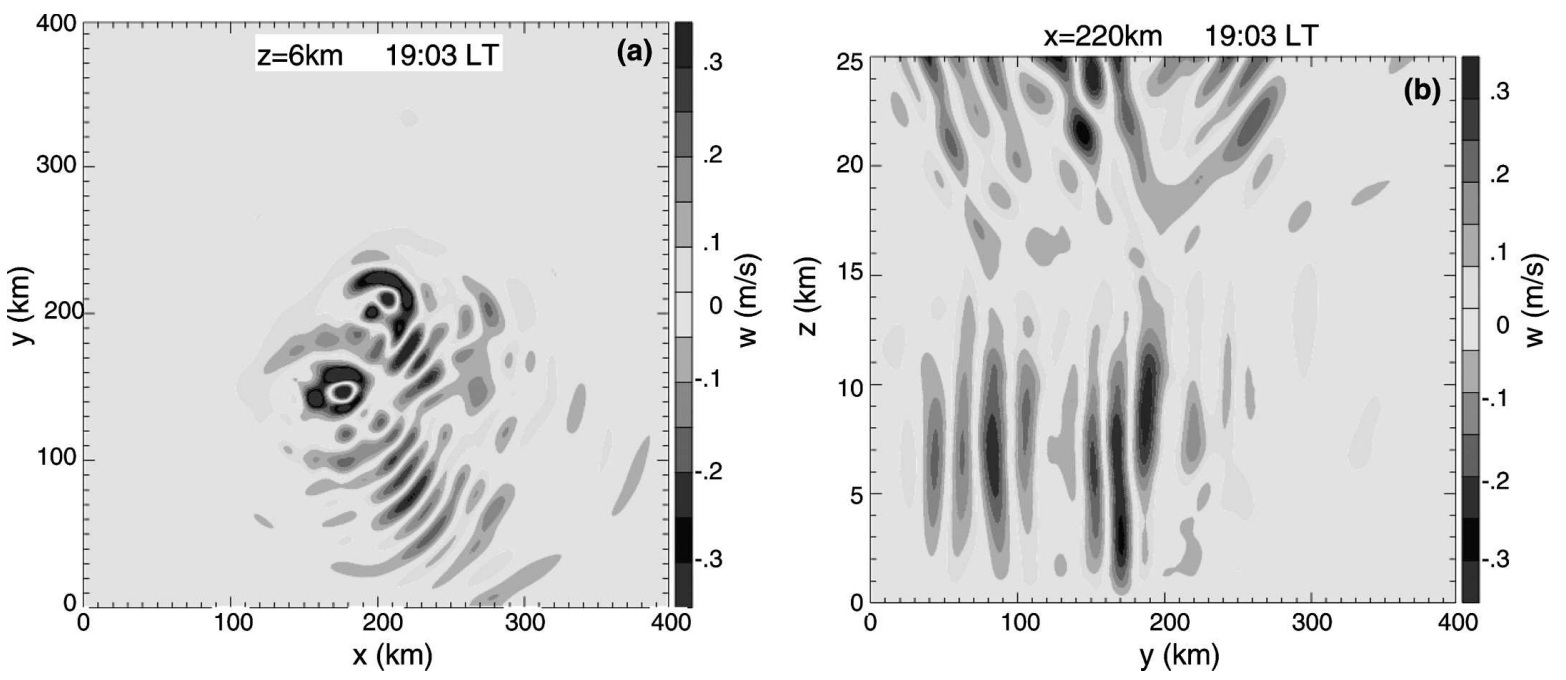

FIG. 2. Vertical velocity at 1903 local time (LT) in the model when the forcing is no longer active and only unforced wave motions remain. (a) Horizontal cross section at 6-km altitude. Trapped southeastward-propagating wave motions are apparent more than $\sim 100$ $\mathrm{km}$ from their source. (b) Vertical cross section in the $y-z$ plane at $x=220 \mathrm{~km}$ to illustrate the vertical structure of the trapped waves. The waves are partially trapped by internal reflection in the troposphere and propagate horizontally with only weak attenuation.

trum of waves in the troposphere in the spaces between strong vertical velocities occurring within the forced updrafts, the latter must be masked prior to taking the Fourier transform. We chose a height-dependent mask, applying $\left|w^{\prime}\right|<w_{\max }(z)$ with an empirical profile $w_{\max }(z)$ chosen to be just larger than the largest wave perturbations at each height. The value of $w_{\max }(z)$ varies smoothly from $0.375 \mathrm{~m} \mathrm{~s}^{-1}$ at $3 \mathrm{~km}$ to $0.85 \mathrm{~m} \mathrm{~s}^{-1}$ at $12 \mathrm{~km}$. Figure 3 shows the power spectra computed from the space-time Fourier cospectra of the masked vertical velocities in the troposphere, $3-12 \mathrm{~km}$ for the Alexander et al. (2004) case shown in the top panel in Fig. 1. These spectra are also averaged over each of four quadrants and plotted versus horizontal wavenumber and frequency. The dashed lines are lines of constant phase speed as marked. The features in these spectra show the combined effects of the wave generation mechanisms and trapping. As was shown in Fig. 1 and will be described further below, the low-level winds have no discernable effect on the vertically propagating wave spectrum and have only a weak effect on the wave trapping, whereas both are very sensitive to the uppertroposphere wind shear.

The high phase speed lobes in each panel in Fig. 3 are associated with a tropospheric vertical wavelength $\lambda_{z}=$ $2 \Delta D \sim 33 \mathrm{~km}$, the response expected from deep convective heating $D \sim 16.5 \mathrm{~km}$. Figure 4 shows the distribution of heating depths input to the model, which in fact peak at $16 \mathrm{~km}$. The second lobe with phase speeds $\sim 20-40 \mathrm{~m} \mathrm{~s}^{-1}$ is likely associated with enhanced wave trapping, as will be explained in the next section. The lowest phase speed lobe in the NE quadrant is a rem- nant of the imperfect masking of the forced updrafts, not trapped waves.

The peaks in the trapped wave spectrum from the first $4 \mathrm{~h}$ of the simulation occur for southeastwardpropagating waves with $(\omega, k)=\left(3.2 \mathrm{~h}^{-1}, 2.3 \times 10^{-2}\right.$ $\left.\mathrm{km}^{-1}\right)$ and $\left(0.8 \mathrm{~h}^{-1}, 1.0 \times 10^{-2} \mathrm{~km}^{-1}\right)$. Using the nonhydrostatic dispersion relation, both of these modes have a ground-relative horizontal group speed of 25 $\mathrm{m} \mathrm{s}^{-1}$ to the southeast. Such trapped waves generated by the isolated island convection in the early afternoon (Alexander et al. 2004) would travel $225 \mathrm{~km}$ southeastward in the 2.5 -h time interval between the early island convection and the later development of the squall line to the southeast (Alexander et al. 2004), placing these wave perturbations in the right place at the right time and with the right orientation to aid in the organization and initiation of the squall line. We estimate the peak tropospheric vertical wind perturbations due to these trapped waves to have a local amplitude of $\sim 1-1.5$ $\mathrm{m} \mathrm{s}^{-1}$. This is an estimate because of the remaining uncertainty in the magnitude of the latent heating that forces the waves in the model (Alexander et al. 2004). We next investigate the spectral selectivity of wave trapping with linear theory.

\section{Wave transmission and reflection}

To investigate wave trapping and its spectral and directional preference in our model, we examine wave transmission and reflection with linear theory. The propagation of internal waves in a nonuniformly stratified fluid with vertically varying background flow is often assessed by ray theory (Lighthill 1978). This pre- 

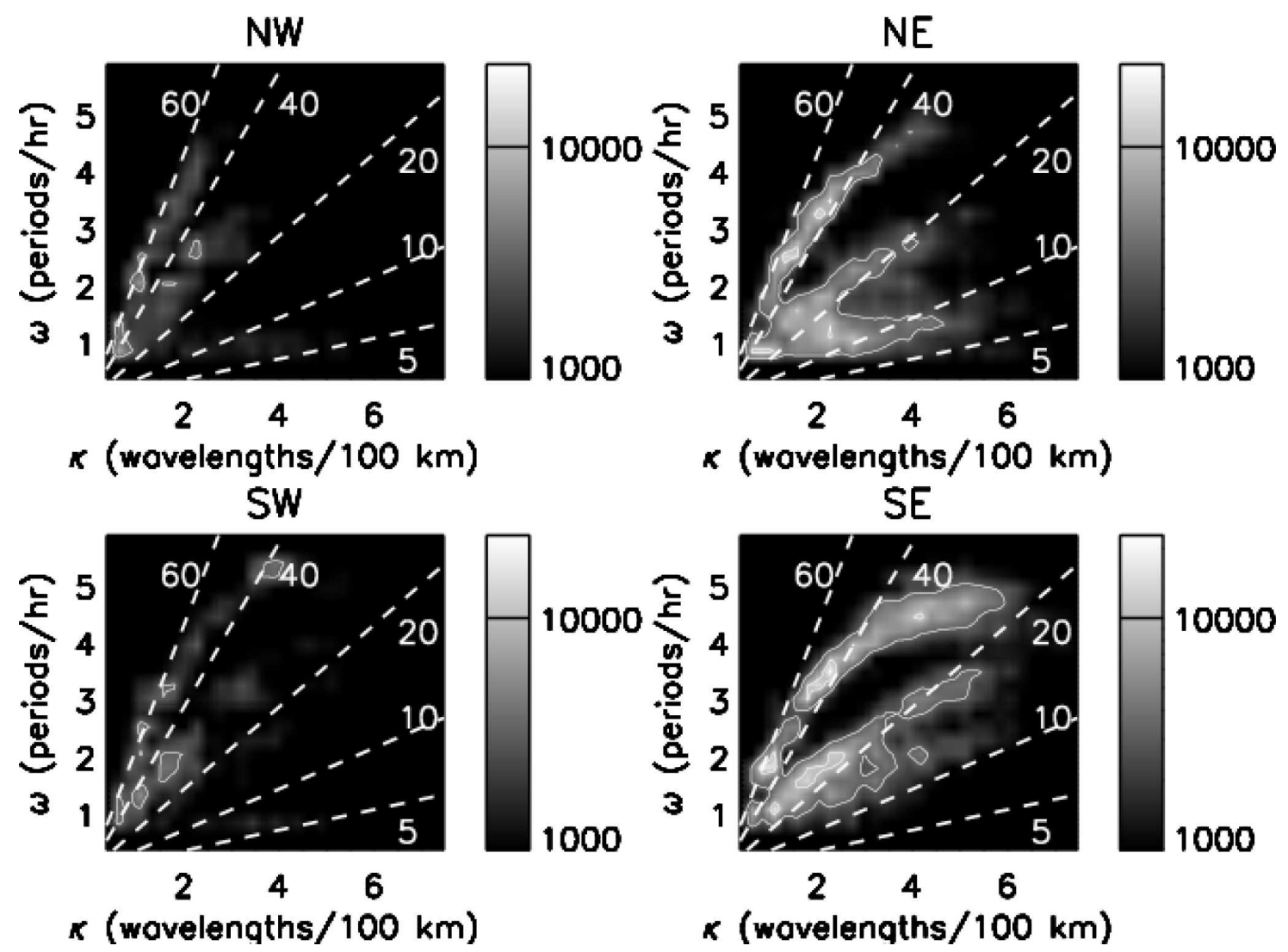

FIG. 3. Vertical velocity power spectra in the troposphere after masking of forced updrafts as described in the text. The spectral units are $\left(\mathrm{m}^{2} \mathrm{~s}^{-2}\right)(\Delta \omega)^{-1}(\Delta \kappa)^{-1}$ and are shown as functions of horizontal wavenumber $\kappa$ [in cycles $(100 \mathrm{~km})^{-1}$ ] and ground-based frequency $\omega$ [in cycles per hour $(\mathrm{cph})]\left(\Delta \omega=6.9 \times 10^{-5}\right.$ cycles s$^{-1}$ and $\Delta \kappa=2.5 \times 10^{-6} \mathrm{cycles} \mathrm{m}^{-1}$.) NE, NW, SW, and SE refer to the four quadrants of wave propagation direction, northeastward, northwestward, southwestward, and southeastward, respectively. Dashed lines are constant phase speed labeled in $\mathrm{m} \mathrm{s}^{-1}$.

dicts the path followed by a quasi-monochromatic small-amplitude wave packet, provided that the background varies slowly compared with the vertical wavelength [Wentzel-Kramer-Brillouin (WKB) approximation]. Two often-invoked heuristics follow from this analysis: 1) internal waves asymptotically approach a critical level where the background wind speed matches the horizontal phase speed of the waves and 2) the waves reflect from a level where their Doppler-shifted frequency matches the background buoyancy frequency.

As with any approximate theory, caution must be taken when applying the above. Internal waves can penetrate beyond reflection and critical levels, as classically defined in two dimensions if the background veers with height (Shutts 1995, 1998) or varies in time (Eckermann 1997; Buckley et al. 1999) or if the wave packets are of sufficiently large amplitude (Sutherland 2000).

Even for small amplitude waves in steady, twodimensional flows, transmission across a reflection level can occur if the depth of the region over which the waves are evanescent is sufficiently small. This circumstance was examined analytically by Sutherland and Yewchuk (2004) for the case of waves propagating in an unsheared background flow with stratification prescribed by piecewise-constant buoyancy frequency. More recently, Nault and Sutherland (2006, manuscript submitted to Phys. Fluids) computed the structure of small amplitude waves propagating in an arbitrarily

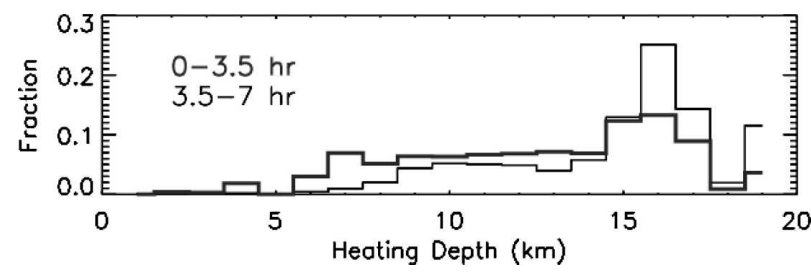

FIG. 4. Heating magnitude-weighted distribution of heating depths input into the model. Two distributions are shown for hours 0-3.5 (gray) and 3.5-7 (black) of the Alexander et al. (2004) simulation time. The distributions peak at a heating depth of $16 \mathrm{~km}$. 
specified profile of background stratification, $N^{2}(z)$, and mean flow, $U(z)$.

To examine the spectral dependence of wave transmission and reflection occurring over vertical distances much smaller than the pressure scale height, we proceed by solving the two-dimensional Boussinesq (Spiegel and Veronis 1960) form of the Taylor-Goldstein equation, which includes nonhydrostatic effects:

$$
\phi_{z z}-k^{2}\left[-\frac{N^{2}}{(\omega-k U)^{2}}-\frac{U_{z z}}{\omega-k U}+1\right] \phi=0 .
$$

Subscript $z$ denotes vertical derivatives, $\phi(z)$ is the streamfunction amplitude, and $k$ and $\omega$ are prescribed values, respectively, of horizontal wavenumber and ground-based frequency. As we are primarily interested in wave reflection, we focus our examination upon waves with $\omega$ and $k$ chosen so that they do not encounter a critical level. That is, we restrict the phase speed $\omega / k$ so that nowhere does it equal $U$ over the domain of integration; therefore (1) is not singular. In these regions of spectral space, the wave transmission is assumed to be zero but flagged with a value of -1 to distinguish from perfect reflection.

At some vertical level, $z_{i}, \phi_{1}$ is specified as the superposition of an incident wave propagating in one direction and a guess at the amplitude and phase of a reflected wave that propagates in the opposite direction. Integrating (1) to a vertical level $z_{f}$ gives the value of $\phi_{1}\left(z_{f}\right)$ and its derivative. The procedure is repeated first by specifying $\phi_{2}$ as the superposition of the same incident wave with a different guess as to the structure of the reflected wave and then by integrating (1) to find $\phi_{2}\left(z_{f}\right)$.

Invoking causality, one then determines a linear combination of $\phi_{1}\left(z_{f}\right)$ and $\phi_{2}\left(z_{f}\right)$ that ensures that the resulting transmitted wave propagates solely in the same direction as the incident wave. Using this linear combination and normalizing, $\phi$ can be constructed everywhere between $z_{i}$ and $z_{f}$ so that it describes the structure of an incident wave with amplitude $A_{i}$ that partially reflects with amplitude $A_{r}$ and partially transmits with amplitude $A_{t}$.

Here partial transmission occurs as a consequence of wave propagation in a nonuniformly stratified fluid while being Doppler-shifted by background winds. Related to the corresponding quantity in optics and quantum mechanics, we define the transmission coefficient to be $T \equiv 1-\left|A_{r} / A_{i}\right|^{2}$, which represents the fraction of wave action that is transported to $z_{f}$ from waves incident at $z_{i}$.

Figure 5 shows transmittance for the no-wind case (the simulation shown in the bottom panels in Fig. 1)

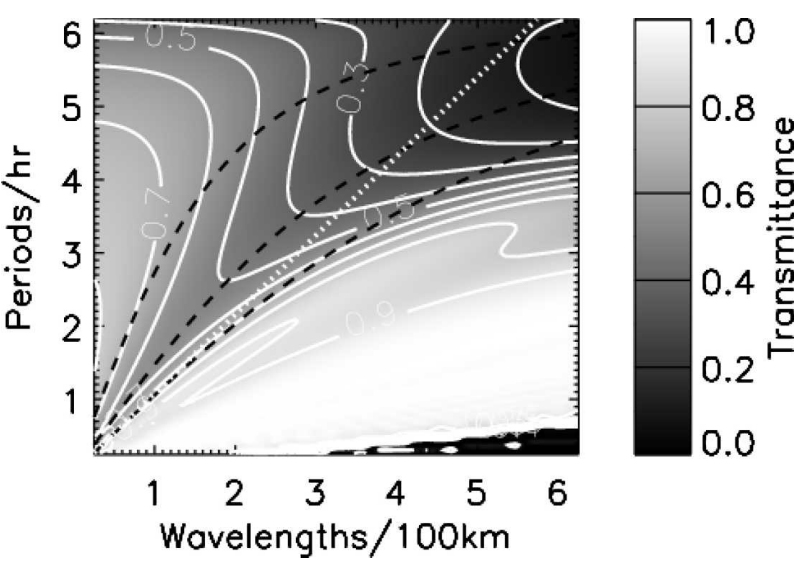

FIG. 5. Transmittance for the no-wind case as a function of horizontal wavenumber and frequency with lines of constant vertical wavelengths, 17, 24, and $48 \mathrm{~km}$, overplotted (dashed) for buoyancy frequency $N=0.011 \mathrm{~s}^{-1}$ representing the value in the tropospheric duct. A line of constant phase speed $=30 \mathrm{~m} \mathrm{~s}^{-1}$ is also overplotted (dotted) for reference.

versus horizontal wavenumber $k$ and frequency $\omega$. A lobe of low transmission occurs centered along a line of constant tropospheric vertical wavelength of $24 \mathrm{~km}$ (center dashed line). This represents a wave with approximately twice the wavelength of the depth of the stability duct, marked by a region of low stability in the upper troposphere between 12 and $15 \mathrm{~km}$. We also show lines of constant vertical wavelength of $48 \mathrm{~km}$, corresponding to the $1 / 4$ wavelength ducted mode described in Lindzen and Tung (1976; upper dashed line) and, also, $17 \mathrm{~km}$ corresponding to the depth of the troposphere and the approximate depth of the strongest latent heating cells (lower dashed line). Plotted for reference is a line of constant phase speed $c=30 \mathrm{~m} \mathrm{~s}^{-1}$, which roughly corresponds to the minimum in transmittance.

To show the additional effects of the winds on wave transmission and reflection, we compute transmittance versus phase speed averaged over horizontal wavelengths prominent in the model $(20-200 \mathrm{~km})$ in Fig. 6 . The no-wind case is shown with the dotted line. Wind effects along the diagonal coordinates SW-NE are shown with the gray line and NW-SE with the solid black line. The upper-tropospheric wind shear causes critical level removal of low phase speed, westwardpropagating waves, reduces transmission for eastwardpropagating waves, and increases transmission for westward-propagating waves that do not have critical levels.

These results suggest that the eastward-propagating trapped waves in our model troposphere are trapped via internal reflection, which is most effective at higher phase speeds. The directional preference of trapping is opposite to that inferred by Shige (2001) and does not 

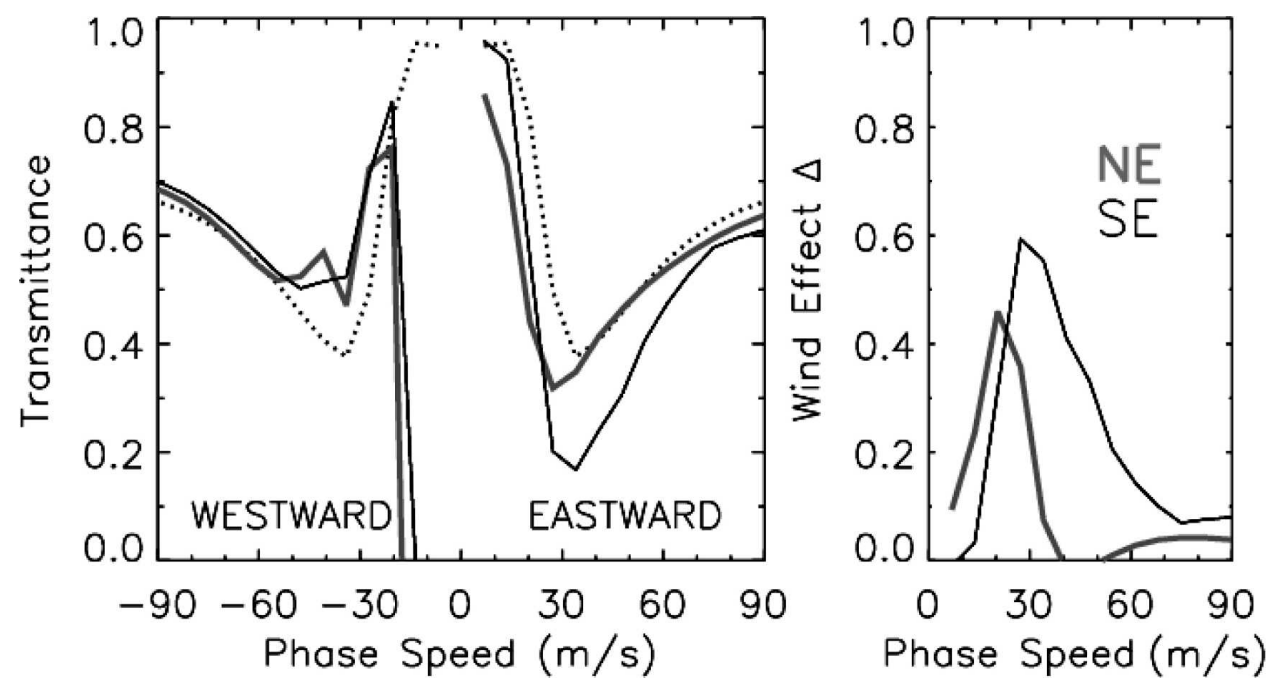

FIG. 6. (left) The transmission coefficient vs phase speed averaged over horizontal wavelengths dominant in the model $(20-200 \mathrm{~km}$ ) for the no-wind case (dotted) and full-wind case averaged along the SW-NE line (gray) and along the NW-SE line (solid). Positive phase speeds have an eastward component, and negative westward. These average transmission coefficients are somewhat sensitive to the range of horizontal wavelength included in the average, resulting in errors of $\pm 5 \%$. (right) The effect of wind reflection ( $\Delta$ defined in the text) vs phase speed for NE (gray) and SE (solid) propagating waves. Errors in $\Delta$ are $\pm 10 \%$.

occur via the overreflection mechanism that they hypothesized. The differences between our results may be explained by differences in the phase speeds and directions of the waves generated in the two models. In the two-dimensional squall line case modeled by Shige (2001), there is a well-known preference for westward wave generation opposite to the direction of the fastmoving eastward storm propagation (e.g., Fovell et al. 1992). Likely, there is an absence of eastward-propagating waves generated in their model that might be trapped via total internal reflection. The wave generation in our model is instead associated with short-lived, slow-moving, deep heating cells that generate waves in the troposphere with fast phase speeds relative to the ground. (The slow phase speed waves in our model are generated above the upper-tropospheric shear layer.) Only a small fraction of the tropospheric wave spectrum has a critical level in the upper troposphere. Instead, the relatively high phase speeds in our model lead to a preference for the reflection of waves propagating opposite to the upper-level winds via the total internal reflection mechanism.

The effects of wind reflection can be isolated by computing

$$
\Delta=1-\frac{T_{U}}{T_{n}}
$$

where $T_{n}$ is the transmittance for the no-wind case and $T_{U}$ the transmittance with wind effects included. The fractional wind reflection effect $\Delta$ is shown in the right panel in Fig. 6 for both northeast- and southeastpropagating waves. The wind effects enhance wave reflection for northeast-propagating waves with phase speeds $20-30 \mathrm{~m} \mathrm{~s}^{-1}$, but the effect is most pronounced for southeast-propagating waves with phase speeds 25 $60 \mathrm{~m} \mathrm{~s}^{-1}$, an important range of phase speeds in our model of waves generated by deep convection. These linear wave transmission/reflection calculations appear to explain the preference for the trapping of SEpropagating waves observed in the model.

\section{Quantifying the obstacle effect mechanism of wave generation}

The three experiments shown in Fig. 1 suggested that the upper-level wind shear has a pronounced effect on the stratospheric wave spectrum, most notably through generation of low phase speed waves via an obstacletype effect of wave generation but also through wind filtering effects such as critical level interactions and wave reflection processes. To separate the wave generation and wind filtering effects, we filter the spectrum from the no-wind case with the wind transmittance effects show in Fig. 6. We then subtract this spectrum from the full-wind case spectrum, thus isolating the wave generation effects of the shear on the stratospheric wave spectrum.

Figure 7 shows momentum flux spectra versus phase 

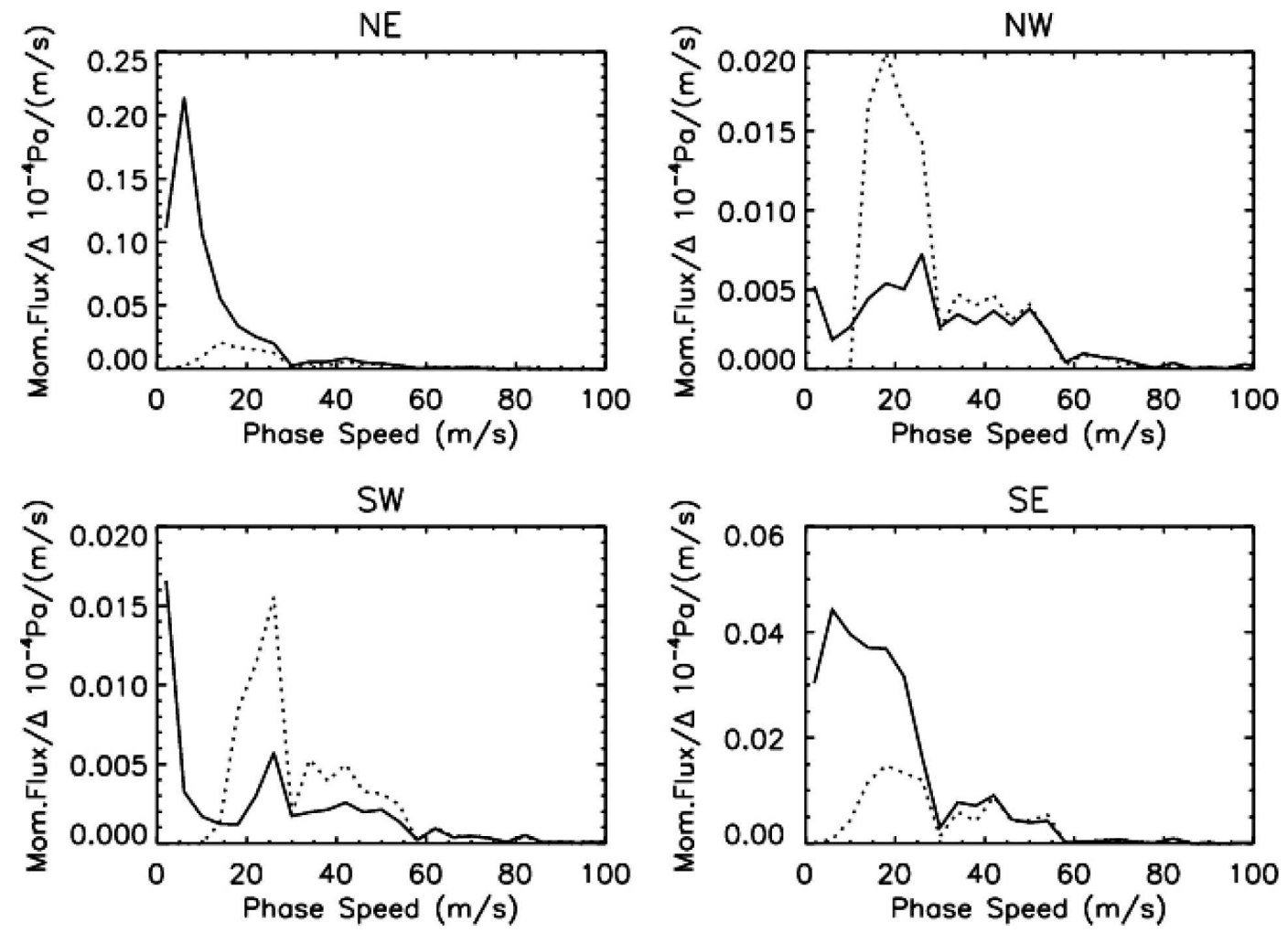

FIG. 7. Momentum fluxes vs phase speed from the model run with full winds (solid) and that run with no wind filtered with the wave transmittances shown in Fig. 5 (dotted). Note the change in ordinate scale between panels (the fluxes are the largest by far in the NE propagation direction).

speed integrated over the four quadrants of propagation direction. The solid line is the flux from the fullwind model, which is the same as that reported in $\mathrm{Al}-$ exander et al. (2004). The dotted line shows the flux from the no-wind model times the factor $T_{U} / T_{n}$, which accounts for the wind reflection effects and removes waves that would have critical levels. Except for critical level removal, the wind filtering effects appear relatively small in this comparison but are included for completeness.

Although differences for westward-propagating waves (NW and SW) with phase speeds $10-30 \mathrm{~m} \mathrm{~s}^{-1}$ look large in Fig. 7, it should be noted that the varying ordinate scale between panels exaggerates these effects because the westward fluxes are relatively weak. The full-wind model westward fluxes at $10-30 \mathrm{~m} \mathrm{~s}^{-1}$ are weaker than the wind-filtered no-wind model because wave refraction to short vertical scales coupled with model diffusion removes portions of the spectrum beyond the phase speeds that encounter critical levels (see also Alexander and Holton 1997; Beres et al. 2002). The primary differences occur for northeastwardpropagating waves at low phase speeds, as seen in the difference plot in Fig. 8.
Figure 8 shows the differences between the solid and dotted lines in Fig. 7 representing wave fluxes generated via the upper-troposphere obstacle effect. Fluxes in the northeast direction with phase speeds of 5-10 $\mathrm{m} \mathrm{s}^{-1}$ are by far the largest, with a smaller fraction in the southeast direction and additional very small fluxes at near-zero phase speeds in the northwest and southwest quadrants. The phase speeds of these obstacle effect waves approximately match the speeds and directions of the latent heating cells that peak at $5 \mathrm{~m} \mathrm{~s}^{-1}$ in azimuth $70^{\circ}$ but form a distribution of phase speeds $\sim 0-15 \mathrm{~m} \mathrm{~s}^{-1}$ in a range of azimuths from southeast to north (Fig. 9).

\section{Parameterization of convectively generated gravity waves}

The properties of waves generated by convection are known to be sensitive to the upper-troposphere winds and to wind-relative motion of the convective heating cells. To apply a parameterization, one must reduce the potentially complex distribution of latent heating cell properties within a grid-box area to a few parameters. In a Whole Atmosphere Community Climate Model 

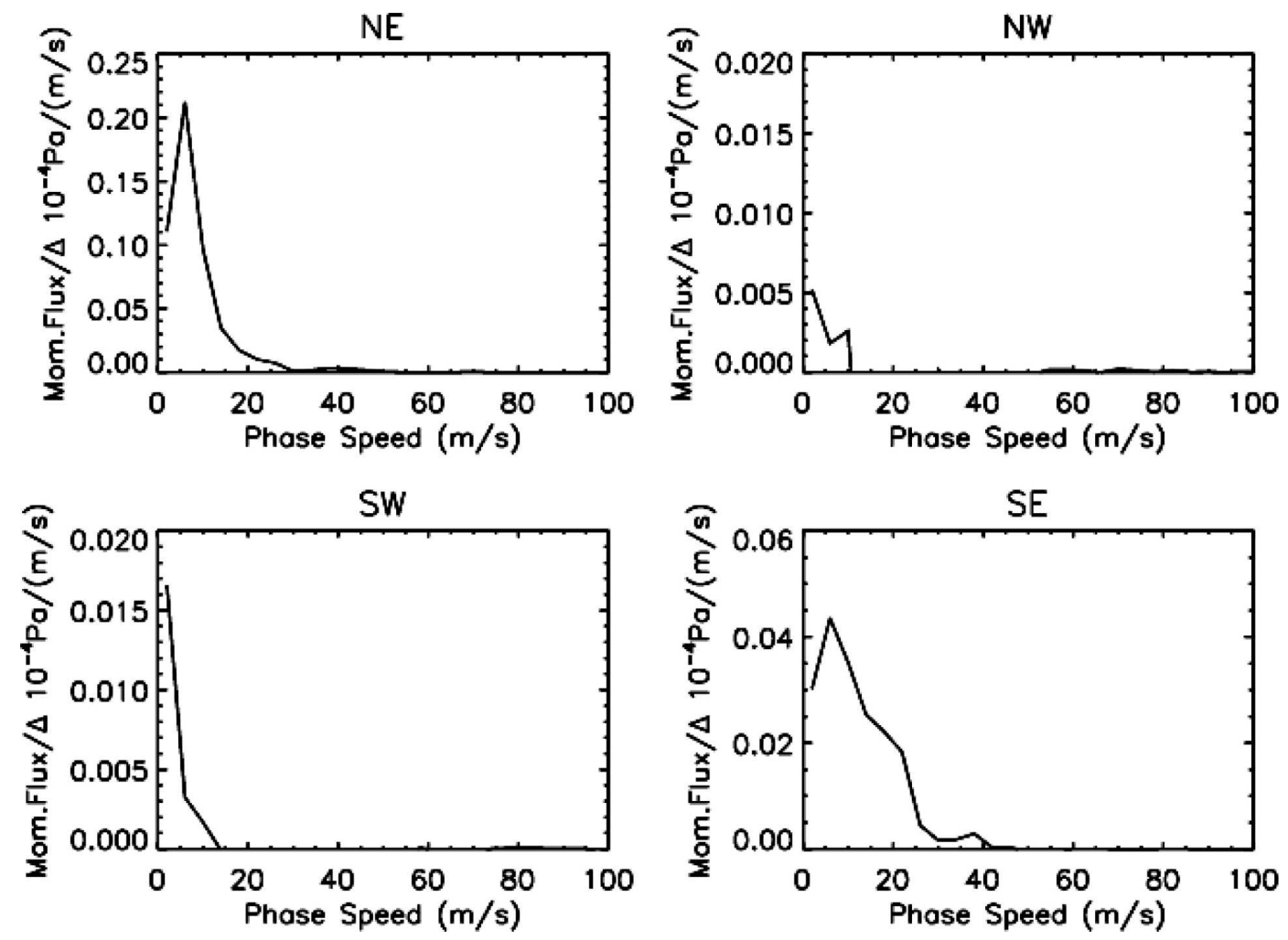

FIG. 8. Differences between the full-wind and filtered no-wind cases (solid minus dashed lines in Fig. 6) showing the momentum flux vs phase speed associated with wave generation via the obstacle effect. Note the change in ordinate scale between panels. Fluxes are largest by far in the NE propagation direction.

(WACCM) study (Beres et al. 2005) the parameterization was applied by assuming that the motion of the heating cells was equal to the large-scale vector wind at $700 \mathrm{mb}$. The horizontal scales and depths of the heating were set to single parameters, and the time scales are represented by a broad red frequency spectrum. Wave reflection effects were neglected. This approach generates a realistic and broad spectrum of gravity wave phase speeds that will be important to the most prominent tropical middle atmosphere wind oscillations like the quasi-biennial oscillation (QBO) and seminannual oscillation (SAO). The Beres et al. (2005) application did not include wave forcing associated with upperlevel wind shear relative to the motion of the heating cells, the so-called obstacle effect.

Although a formulation for wave generation via the obstacle effect is included in Beres et al. (2004), the practical application has large uncertainties. Chun and Baik (1998, 2002) also formulated a parameterization for wave generation via the obstacle effect, and it has similar uncertainties. The main issue is that the heating depth is not a single parameter, as must be assumed for parameterization, but a distribution of depths, and the relevant wind speed flowing over the heat cell obstacle can depend sensitively on the depth of the cell. The momentum fluxes generated via this obstacle effect mechanism are quite sensitive to the wind speeds relative to the heating cell (Beres et al. 2004), and the relevant wind speed will depend on how deeply the heat cell penetrates into the overlying shear zone. If the shear is strong, these uncertainties are large, so the resulting uncertainties in the fluxes will be large. For Chun and Baik (1998), the uncertainty is embodied in the simple parameters $\bar{U}_{2}$ and $a$ for wind and heating depth since it is only the stationary waves relative to the heating that are included in their parameterization. In

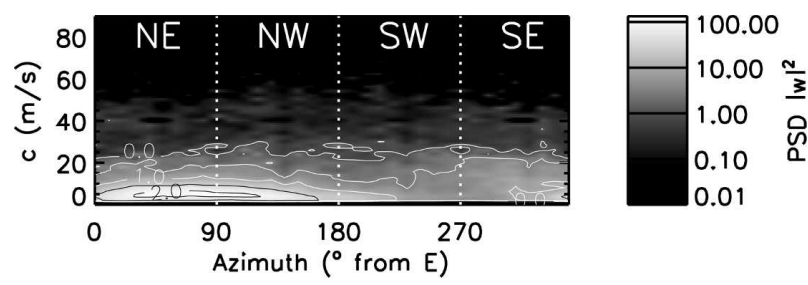

FIG. 9. Convective updraft spectrum vs propagation azimuth and phase speed averaged over altitudes $12.25-15.25 \mathrm{~km}$. The distribution of power shows average speeds and directions of the deep convective cells that move primarily NE at $5 \mathrm{~m} \mathrm{~s}^{-1}$. 

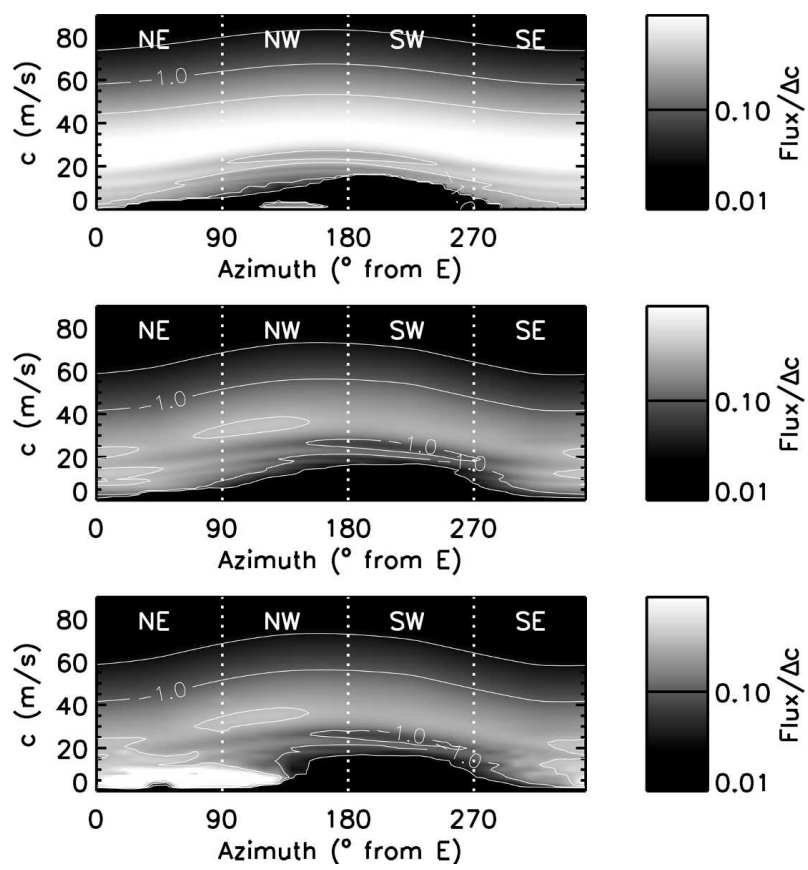

FIG. 10. Wave momentum flux vs propagation direction and phase speed for three different sets of parameterization settings described in Table 1: (top) case W uses the Beres et al. (2005) settings that were applied in WACCM, (middle) case D uses settings modified to include the known properties of the resolved convection in the radar domain and wave reflection effects, and (bottom) cases D plus $\mathrm{S}$ adds the obstacle effect wave generation (case S) to case D. Flux units are $10^{-6} \mathrm{~kg} \mathrm{~m}^{-2} \mathrm{~s}^{-1}$.

the Beres et al. (2004) approach, which describes both stationary and nonstationary wave generation, an additional issue of the momentum flux in this stationary component relative to the momentum flux in the nonstationary waves would add complexity, requiring two separate poorly constrained tuning parameters. Although this approach might be more realistic, Beres et al. (2005) chose to omit this complexity because of the large uncertainties.

Here we examine the predicted momentum flux spectrum from the Beres et al. (2004) parameterization using settings relevant to the Darwin area on 17 November 2001. Figure 10 shows the momentum flux versus phase speed and propagation direction for three sets of parameterization settings described in Table 1. These should be compared to the top panel in Fig. 1. Case $\mathrm{W}$ uses the parameterization settings applicable to the assumptions applied in Beres et al. (2005). The heating cells are assumed to travel with the speed and direction $\left(u_{c}, v_{c}\right)$ of either the 700-mb background wind or this value minus $10 \mathrm{~m} \mathrm{~s}^{-1}$ if the speed exceeds 10 $\mathrm{m} \mathrm{s}^{-1}$. The cell depth is computed from the half-sine wave fit to the average heating profile in the domain and the heating rate is the average over the domain. The mean wind in the heating region $\left(U_{h}, V_{h}\right)$ is the average background wind over the depth of the heating. These settings describe the nonstationary wave generation.

The middle panel in Fig. 10 shows case D parameter settings, which have been modified to reflect the known details of the resolved heating in the DAWEX radar domain. Wave reflection from our calculations in section 3 is also included by multiplying the spectrum predicted by the parameterization by the transmission coefficients computed for the DAWEX model background winds and stability profiles in section 3 . The primary difference between cases $\mathrm{W}$ and $\mathrm{D}$ are the effects of wave reflection, with the motion of the heating cells $\left(u_{c}, v_{c}\right)$ having additional small effects. Other differences are negligible. As for case $\mathrm{W}$, case D describes only the nonstationary wave generation.

Case $\mathrm{S}$ describes stationary wave generation via the obstacle effect. We use the shape of the spectrum of tropospheric $w$ (Fig. 9) to define the shape of a distribution of cell motions $\left(u_{c}, v_{c}\right)$. The distribution, normalized to unity, then describes the fractional distribution of momentum flux versus phase speed for the case $\mathrm{S}$ waves that are stationary relative to the heat cell motions $\left(u_{c}, v_{c}\right)$. The background wind speed and direction over the top of the obstacle is also assumed to be the distribution of wind speeds from $12-$ to $15-\mathrm{km}$ altitude.

TABLE 1. Parameterization settings.

\begin{tabular}{llll}
\hline \hline & \multicolumn{1}{c}{ Case } \\
\cline { 2 - 3 } & \multicolumn{1}{c}{$\mathrm{W}$} & \multicolumn{1}{c}{$\mathrm{D}$} & \multicolumn{1}{c}{$\mathrm{S}$} \\
\hline Description & Beres et al. $(2005)$ & Modified DAWEX & Stationary waves \\
Heating depth & $12 \mathrm{~km}$ & $12.6 \mathrm{~km}$ & $12.6 \mathrm{~km}$ \\
Cell speed $\left(u_{c}, v_{c}\right)$ & $(-1.47,0.7) \mathrm{m} \mathrm{s}^{-1}$ & $(1.7,4.7) \mathrm{m} \mathrm{s}^{-1}$ & Distribution \\
Wind $\left(U_{h}, V_{h}\right)$ & $(-5.19,1.48) \mathrm{m} \mathrm{s}^{-1}$ & $(-5.19,1.48) \mathrm{m} \mathrm{s}^{-1}$ & $(-5.0,-5.0)-(-14.8,-2.1) \mathrm{m} \mathrm{s}^{-1 *}$ \\
Heating rate & $5.94 \mathrm{~K} \mathrm{day}^{-1}$ & $5.94 \mathrm{~K} \mathrm{day}^{-1}$ & $5.94 \mathrm{~K} \mathrm{day}^{-1}$ \\
Wave reflection & Neglected & Included & n/a \\
\hline
\end{tabular}

* For the stationary waves, this is the wind at the top of the obstacle. 
This roughly describes the variations in cell penetration depth into the $12-15-\mathrm{km}$ shear zone. Since these waves are generated above the upper-tropospheric shear zone, wave reflection effects are not applied to these waves generated via the obstacle effect.

The bottom panel in Fig. 10 shows the sum of stationary and nonstationary wave fluxes, cases S plus D, which can be compared to the DAWEX model results in the top panel in Fig. 1. Differences in the magnitude of the fluxes remain, and these will likely remain somewhat tunable in global circulation model (GCM) applications of the parameterization. We focus here on the shape of the spectrum and on the relative magnitude of the stationary and nonstationary wave components. The comparison shows that the modified parameterization captures many of the important features of the model fluxes quite well considering the gross simplifications required for the parameterization. The differences include the following. (i) The critical level filtering extends to larger phase speeds in the model, carving out a larger arc of zero fluxes. The cause is the numerical diffusion in the model, which effectively removes short vertical wavelength waves before they reach critical levels. (ii) The width of the phase speed distribution is somewhat broader in the parameterization than in the model, and the nonstationary wave phase speed peak occurs at higher values in the parameterization ( $\sim 25$ versus $\sim 20 \mathrm{~m} \mathrm{~s}^{-1}$ ). (iii) The magnitudes of the parameterized fluxes are somewhat larger, as expected, $20 \%$ larger for northeastward-propagating waves. Nonlinear effects are explored further in the next section.

\section{Quantifying nonlinear effects}

Song et al. (2003) called attention to nonlinear effects in gravity wave generation by convection. They compared wave spectra generated in a two-dimensional cloud-resolving model to waves generated in a linearized model forced with the latent heating from the full simulation. Their results showed that the linear model generated very similar wave properties to those generated in the full model, but the linear assumption tends to overpredict the wave fluxes, particularly at slower phase speeds. The wave amplitudes are apparently overpredicted because heat and momentum flux convergence terms omitted in a linear model partially cancel the heating in a nonlinear model.

Our model is nonlinear, so, if the magnitude of our input latent heating is accurate, the nonlinear terms missing in the Song et al. (2003) study will be included. However, in Alexander et al. (2004), we found that our input heating rates were likely a factor of 3-5 times too small when we compared the waves to observational constraints.

We therefore examine nonlinear corrections to our model by increasing the strength of the heating by factors of 3 and 5 in two additional simulations and compare the results in Fig. 11. The solid line is the same as in Fig. 7, while dotted and dashed curves show wave fluxes for the $3 \times$ and $5 \times$ simulations divided, respectively, by factors of 9 and 25 that would be expected from linear theory (Chun and Baik 1998; Beres et al. 2004). This comparison shows that the wave fluxes in the simulations do not scale linearly over this range of a factor of 5, but nonlinear effects are evident, particularly at the lower phase speeds. Nonlinear effects are essentially absent at phase speeds greater than $\sim 20 \mathrm{~m} \mathrm{~s}^{-1}$. The low phase speed nonlinear effects are in the same sense as those reported in Song et al. (2003), but our effects are very much smaller, likely because their linearization was accomplished by reducing the magnitude of heating by a factor of $10000 \times$ compared to our factors of $3 \times$ and $5 \times$. The linear assumption is, however, a prominent feature of parameterizations (Chun and Baik 1998, 2002; Beres et al. 2004), so the parameterizations should, somehow, be corrected for nonlinear effects.

Our results indicate that a phase-speed-dependent correction factor might be applied to the parameterized spectrum to account for nonlinear effects, but the range of nonlinearity would need to be explored further before proposing a quantitative correction. In lieu of this uncertainty and considering the relatively scarce observational constraints on momentum fluxes, the parameterized fluxes will likely remain a tunable parameter in GCM applications in the near future. We note that mountain wave parameterizations that have been in existence for 20 years still retain a tunable parameter for wave fluxes in GCM applications.

To begin to quantify the differences between wave fluxes in the nonlinear model and the parameterization, we integrate the fluxes over the spectrum. For the model, these represent averages over the 400-km model area and $4 \mathrm{~h}$ of time. For comparison, the parameterization is multiplied by the assumed intermittency factor and a ratio of the WACCM pixel size to the model domain size. Here we quote values for northeastwardpropagating waves. The nonstationary wave fluxes are $0.27 \mathrm{mPa}$ in the baseline DAWEX model and $0.34 \mathrm{mPa}$ in the linear parameterization. The corresponding stationary wave fluxes are $0.19 \mathrm{mPa}$ in the model and 0.22 $\mathrm{mPa}$ in the parameterization.

In the simulations with $3 \times$ and $5 \times$ heating rates, the nonstationary wave component increases approximately linearly while the stationary waves increase less than the linear assumption predicts. For the $1 \times, 3 \times$, 

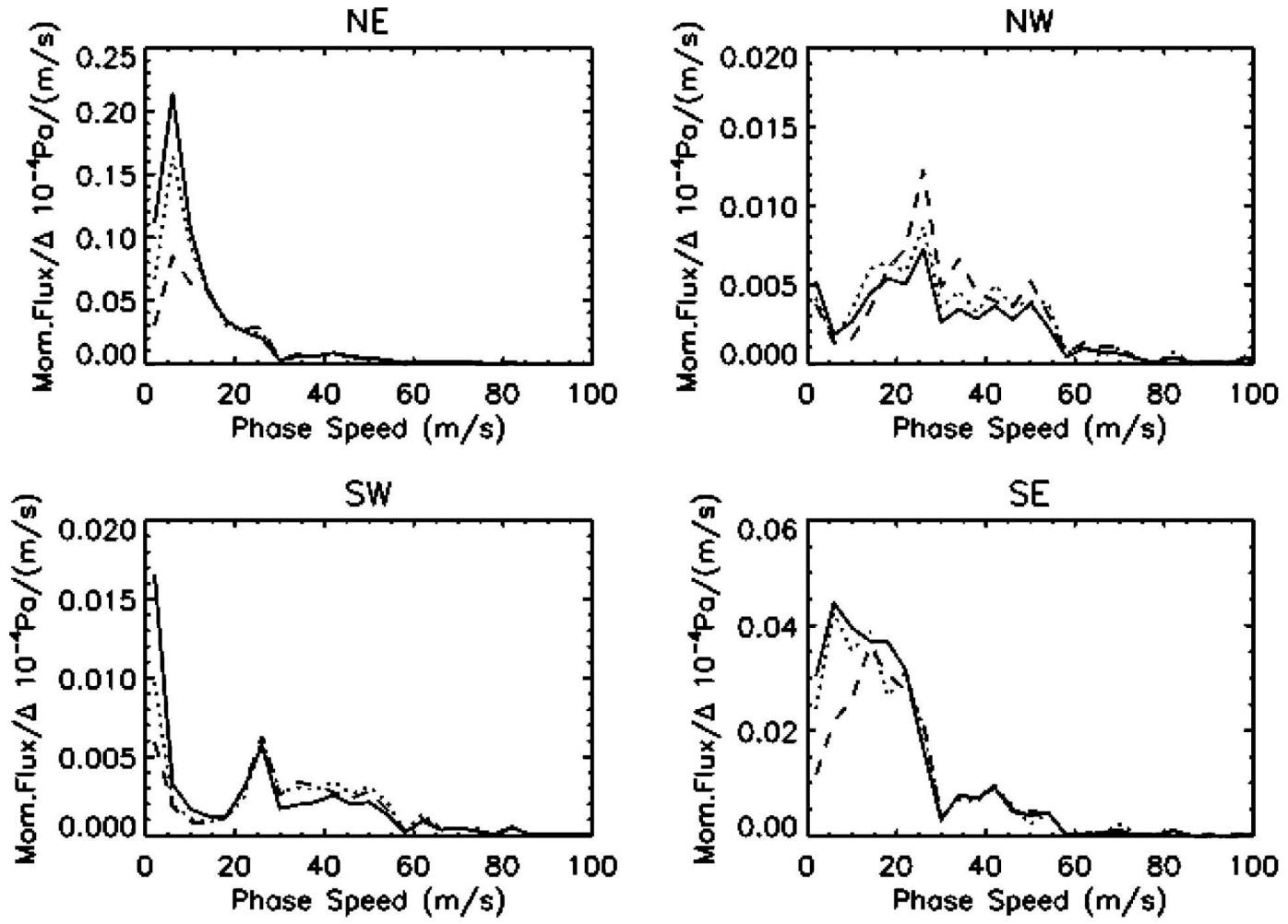

FIG. 11. Stratospheric wave momentum flux spectrum averaged over the four propagation direction quadrants normalized to illustrate nonlinear effects. The solid line is as in Fig. 7. The dotted line shows the wave flux generated in an identical simulation except for input heating magnitudes all multiplied by 3 and wave fluxes divided by 9 . The dashed line is for $5 \times$ input heating amplitudes and wave fluxes divided by 25 .

and $5 \times$ heating cases, the total fluxes in the model are $0.46,3.6$, and $8.1 \mathrm{mPa}$, respectively. (These increases are less than linear since, in the linear assumption, the increase would be by factors of $3^{2}=9$ and $5^{2}=25$.) The deviations from linearity occur in the stationary wave components.

The linear parameterizaton (combined cases $\mathrm{D}$ plus S) predicts that $1 \times, 3 \times$, and $5 \times$ heating cases would generate fluxes of $0.56,5.0$, and $14 \mathrm{mPa}$, respectively. For the most likely $3 \times$ heating rate values based on the DAWEX study (Alexander et al. 2004), the parameterization overpredicts the fluxes by $39 \%$. The agreement would be significantly worse using the case W parameterization.

\section{Summary and conclusions}

According to the comparison in our case study, the parameterization benefits most from the inclusion of two effects: (i) wave reflection and trapping of a fraction of the high phase speed wave energy in the troposphere and (ii) the generation of waves via the obstacle effect. The importance of including these processes in the parameterization will clearly vary depending on the depths of the heating cells and on the wind shear and stability profiles. In this paper, we have described approximate linearized methods that may aid in developing improved versions of the parameterization in future applications, although some additional cases studies would be valuable for this purpose.

In our simulations of waves generated by deep heating, internal reflection traps at least $40 \%$ of eastwardpropagating gravity wave activity at phase speeds greater than $25 \mathrm{~m} \mathrm{~s}^{-1}$. Reflection further traps $\sim 70 \%-$ $80 \%$ of southeastward-propagating waves with phase speed 30-50 $\mathrm{m} \mathrm{s}^{-1}$. Our work shows how these reflection effects can be computed with only knowledge of the large-scale wind and stability profiles, which makes this effect easy to include in convectively generated gravity wave source parameterizations.

An "obstacle effect" is the mechanism responsible for generating waves with low phase speeds and a preference for northeastward propagation in the model stratosphere. The "obstacles" are the deep heating cells that penetrate into the region of strong westward shear at $z \sim 12-15 \mathrm{~km}$. 
Comparison to the Beres (2004) parameterization shows much improved agreement when reflection of high phase speed waves is included as well as stationary waves generated via the obstacle effect. The parameterization, based on linear theory, now compares well to the model's higher phase speed, nonstationary wave flux spectrum but overpredicts the dependence of the low phase speed wave fluxes on the strength of the heating. These increase linearly with heating strength in the parameterization but less than linearly in the model. The Chun and Baik (2002) parameterization is also based on linear theory and neglects nonstationary wave generation, including only the stationary component of the forcing, so it should suffer from similar errors in its dependence on the strength of the heating.

The parameterization of stationary waves in our DAWEX example is also very sensitive to the depth of the heating cell penetration into the upper-level shear zone as well as the motion of the heating cells relative to the ground. These details are not generally known in GCM applications. The parameterization of the stationary wave component therefore remains a challenging problem with many uncertainties.

Acknowledgments. This work was supported by the National Science Foundation Physical Meteorology Program Grant Number 0234230.

\section{REFERENCES}

Alexander, M. J., and J. R. Holton, 1997: A model study of zonal forcing in the equatorial stratosphere by convectively induced gravity waves. J. Atmos. Sci., 54, 408-419.

— , P. T. May, and J. H. Beres, 2004: Gravity waves generated by convection in the Darwin area during the Darwin Area Wave Experiment. J. Geophys. Res., 109, D20S04, doi:10.1029/2004JD004729.

Beres, J. H., 2004: Gravity wave generation by a threedimensional thermal forcing. J. Atmos. Sci., 61, 1805-1815.

- M. J. Alexander, and J. R. Holton, 2002: Effects of tropospheric wind shear on the spectrum of convectively generated gravity waves. J. Atmos. Sci., 59, 1805-1824.

,$- \ldots$, and — 2004: A method of specifying the gravity wave spectrum above convection based on latent heating properties and background wind. J. Atmos. Sci., 61, 324-337.

— , R. R. Garcia, B. A. Boville, and F. Sassi, 2005: Implementation of a gravity wave source spectrum parameterization dependent on the properties of convection in the Whole Atmosphere Community Climate Model (WACCM). J. Geophys. Res., 110, D10108, doi:10.1029/2004JD005504.

Bergman, J. W., and M. L. Salby, 1994: Equatorial wave activity derived from fluctuations in observed convection. J. Atmos. Sci., 51, 3791-3806.

Buckley, G., D. Broutman, J. W. Rottman, and S. Eckermann, 1999: On the importance of weak steady shear in the refraction of short internal waves. Geophys. Res. Lett., 26, 28772880.

Chun, H.-Y., and J.-J. Baik, 1998: Momentum flux by thermally induced internal gravity waves and its approximation for large-scale models. J. Atmos. Sci., 55, 3299-3310.

_ tively forced gravity wave drag for use in large-scale models. J. Atmos. Sci., 59, 1006-1017.

_ I.-S. Song, J.-J. Baik, and Y.-J. Kim, 2004: Impact of a convectively forced gravity wave drag parameterization in NCAR CCM3. J. Climate, 17, 3530-3547.

Clark, T. L., T. Hauf, and J. P. Kuettner, 1986: Convectively forced internal gravity waves: Results from two-dimensional numerical experiments. Quart. J. Roy. Meteor. Soc., 112, 899925.

Dewan, E., and Coauthors, 1998: MSX satellite observations of thunderstorm-generated gravity waves in mid-wave infrared images of the upper stratosphere. Geophys. Res. Lett., 25, 939-942.

Dunkerton, T., 1997: The role of gravity waves in the quasibiennial oscillation. J. Geophys. Res., 102, 26 053-26 076.

Durran, D. R., and J. B. Klemp, 1982: The effects of moisture on trapped mountain lee waves. J. Atmos. Sci., 39, 2490-2506.

—, and _ 1983: A compressible model for the simulation of moist mountain waves. Mon. Wea. Rev., 111, 2341-2361.

_ - M.-J. Yang, D. N. Slinn, and R. G. Brown, 1993: Toward more accurate wave-permeable boundary conditions. Mon. Wea. Rev., 121, 604-620.

Eckermann, S. D., 1997: Influence of wave propagation on the Doppler spreading of atmospheric gravity waves. J. Atmos. Sci., 54, 2554-2573.

Fovell, R., D. Durran, and J. R. Holton, 1992: Numerical simulations of convectively generated stratospheric gravity waves. $J$. Atmos. Sci., 49, 1427-1442.

Giorgetta, M. A., E. Manzini, and E. Roeckner, 2002: Forcing of the quasi-biennial oscillation from a broad spectrum of atmospheric waves. Geophys. Res. Lett., 29, 1245, doi:10.1029/ 2002GL014756.

Holton, J. R., 1972: Waves in the equatorial stratosphere generated by tropospheric heat sources. J. Atmos. Sci., 29, 368-375.

Keenan, T., K. Glasson, F. Cummings, T. S. Bird, J. Keeler, and J. Lutz, 1998: The BMRC/NCAR C-Band Polarimetric (CPOL) radar system. J. Atmos. Oceanic Technol., 15, 871-886.

Lane, T., and M. Reeder, 2001: Modelling the generation of gravity waves by a maritime continent thunderstorm. Quart. J. Roy. Meteor. Soc., 127, 2705-2724.

Lighthill, M. J., 1978: Waves in Fluids. Cambridge University Press, 504 pp.

Lindzen, R. S., 1974: Wave-CISK in the tropics. J. Atmos. Sci., 31, 156-179.

_ , and K.-K. Tung, 1976: Banded convective activity and ducted gravity waves. Mon. Wea. Rev., 104, 1602-1617.

Mapes, B. E., 1993: Gregarious tropical convection. J. Atmos. Sci., 50, 2026-2037.

Marks, C. J., and S. D. Eckermann, 1995: A three-dimensional nonhydrostatic ray-tracing model for gravity waves: Formulation and preliminary results for the middle atmosphere. $J$. Atmos. Sci., 52, 1959-1984.

Matsuno, T., 1966: Quasi-geostrophic motions in the equatorial area. J. Meteor. Soc. Japan, 44, 25-43.

May, P. T., A. R. Jameson, T. D. Keenan, P. E. Johnston, and C. Lucas, 2002: Combined wind profiler/polarimetric radar studies of the vertical motion and microphysical characteristics of tropical sea-breeze thunderstorms. Mon. Wea. Rev., 130, 2228-2239. 
Pfister, L., and Coauthors, 1993: Gravity waves generated by a tropical cyclone during the STEP tropical field program: A case study. J. Geophys. Res., 98, 8611-8638.

Piani, C., D. Durran, M. J. Alexander, and J. R. Holton, 2000: A numerical study of three-dimensional gravity waves triggered by deep tropical convection and their role in the dynamics of the QBO. J. Atmos. Sci., 57, 3689-3702.

Salby, M. L., and R. R. Garcia, 1987: Transient response to localized episodic heating in the tropics. Part I: Excitation and short-time near-field behavior. J. Atmos. Sci., 44, 458-498.

Shige, S., and T. Satomura, 2001: Westward generation of eastward-moving tropical convective bands in TOGA COARE. J. Atmos. Sci., 58, 3724-3740.

Shutts, G. J., 1995: Gravity-wave drag parameterization over complex terrain: The effect of critical level absorption in directional wind shear. Quart. J. Roy. Meteor. Soc., 121, 1005-1021.

_, 1998: Stationary gravity-wave structure in flows with directional wind shear. Quart. J. Roy. Meteor. Soc., 124, 14211442.

Song, I.-S., H.-Y. Chun, and T. P. Lane, 2003: Generation mechanisms of convectively forced internal gravity waves and their propagation to the stratosphere. J. Atmos. Sci., 60, 19601980.
Spiegel, E., and G. Veronis, 1960: On the Boussinesq approximation for a compressible fluid. Astrophys. J., 131, 442-447.

Sutherland, B. R., 2000: Internal wave reflection in uniform shear. Quart. J. Roy. Meteor. Soc., 126, 3255-3287.

- and K. Yewchuk, 2004: Internal wave tunnelling. J. Fluid Mech., 511, 125-134.

Tsuda, T., M. V. Ratnam, P. T. May, M. J. Alexander, R. A. Vincent, and A. MacKinnon, 2004: Characteristics of gravity waves with short vertical wavelengths observed with radiosonde and GPS occultation during DAWEX (Darwin Area Wave Experiment). J. Geophys. Res., 109, D20S03, doi:10.1029/2004JD004946.

Vincent, R. A., A. MacKinnon, I. M. Reid, and M. J. Alexander, 2004: VHF profiler observations of winds and waves in the troposphere during the Darwin Area Wave Experiment (DAWEX). J. Geophys. Res., 109, D20S02, doi:10.1029/ 2004JD004714.

Wheeler, M., and G. N. Kiladis, 1999: Convectively coupled equatorial waves: Analysis of clouds and temperature in the wavenumber-frequency domain. J. Atmos. Sci., 56, 374-399.

Yang, M.-J., and R. A. Houze Jr., 1995: Multicell squall-line structure as a manifestation of vertically trapped gravity waves. Mon. Wea. Rev., 123, 641-661. 